\title{
A novel method for quantitative height measurement based on an astigmatic optical profilometer
}

\author{
Liao, Hsien-Shun; Huang, Guan-Teng; Tu, Ho-Da; Lin, Ting-He; Hwu, En Te
}

Published in:

Measurement Science and Technology

Link to article, DOI:

10.1088/1361-6501/aadc49

Publication date:

2018

Document Version

Peer reviewed version

Link back to DTU Orbit

Citation (APA):

Liao, H-S., Huang, G-T., Tu, H-D., Lin, T-H., \& Hwu, E. T. (2018). A novel method for quantitative height measurement based on an astigmatic optical profilometer. Measurement Science and Technology, 29(10), [107002 ]. https://doi.org/10.1088/1361-6501/aadc49

\section{General rights}

Copyright and moral rights for the publications made accessible in the public portal are retained by the authors and/or other copyright owners and it is a condition of accessing publications that users recognise and abide by the legal requirements associated with these rights.

- Users may download and print one copy of any publication from the public portal for the purpose of private study or research.

- You may not further distribute the material or use it for any profit-making activity or commercial gain

- You may freely distribute the URL identifying the publication in the public portal 


\title{
A novel method for quantitative height measurement based on an astigmatic optical profilometer
}

\author{
Hsien-Shun Liao ${ }^{1, *}$, Guan-Teng Huang ${ }^{1}$, Ho-Da Tu${ }^{1}$, Ting-He Lin ${ }^{1}$, En-Te Hwu ${ }^{2}$
}

\author{
${ }^{1}$ Department of Mechanical Engineering, National Taiwan University, Taipei, Taiwan R.O.C. \\ ${ }^{2}$ Department of Micro- and Nanotechnology, Technical University of Denmark, Denmark \\ *Corresponding author. \\ E-mail: liaohs@ntu.edu.tw
}

Received xxxxxx

Accepted for publication $\mathrm{xxxxxx}$

Published xxxxxx

\begin{abstract}
Astigmatic detection systems have been used in hybrid systems to produce both contact and noncontact profilometers, which provide advantageous features such as low cost, small laser spot, high bandwidth, and compact size. However, current astigmatic optical profilometers cannot provide quantitative height measurement on a surface consisting of complex materials. In this paper, a novel method called z-axis modulation is proposed to overcome this limitation. A homemade astigmatic optical profilometer was developed, and an analytical process for height calculation was also developed. As demonstrated by the experimental results, z-axis modulation can provide accurate height measurement. Furthermore, optical properties such as reflectivity can also be measured.
\end{abstract}

Keywords: optical profilometer, height measurement, reflectivity

\section{Introduction}

Surface profile and roughness measurements are essential in many industrial fields such as optics, micromechanics, and biomedical materials [1-3]. Profilometers are among the most common tools for measuring surface profiles and can be divided into contact and noncontact types [4-6]. Contacttype instruments such as a stylus profilometer and atomic force microscope (AFM) utilize a sharp tip to scan a surface and provide a high-resolution profile [7,8]. Moreover, the frictional properties of the surface are accessible, because the interaction force between the tip and the sample surface is measurable $[9,10]$. However, the interaction force may cause sample damage and tip wear. Furthermore, measurement accuracy can be reduced by tip contamination during the scanning process. Noncontact profilometers are based on optical methods such as interferometry, focus detection, and pattern projection [11-14]. Without physical contact with the sample, an optical profilometer can achieve fast measurement and avoid sample damage. However, the optical profilometer's spatial resolution on the xy plane is limited by optical diffraction.

To combine the advantages of both contact and noncontact measurements, Hwu et al. proposed a hybrid system based on an astigmatic detection system (ADS) [15]. The key component of the ADS is a commercial DVD pickup head, which has the advantages of compact size, low cost, small laser spot size, and easy alignment. By detecting a cantilever tip, the ADS can perform a high-resolution and 
high-speed AFM [16,17]. The ADS can also be turned into an astigmatic optical profilometer by measuring the sample directly without the cantilever tip [18]. Furthermore, an astigmatic optical profilometer has been optimized for imaging biological samples in a liquid environment $[19,20]$.

In the astigmatic optical profilometer, a laser beam is focused on the sample surface, and a photodetector integrated chip (PDIC) receives the reflective laser beam [15]. Because of the astigmatic effect, the shape of the laser spot on the PDIC varies with the surface height, which can be detected by the focus error signal $U_{F E S}$. Figure 1(b) illustrates the S-curve representing the relationship between $U_{F E S}$ and surface height. Because the middle region of the Scurve is linear, $U_{F E S}$ is proportional to the surface height. When the sample is scanned using the laser, the surface profile can be represented by $U_{F E S}$, as shown in figure 1(a). Because the focused laser only scans on the horizontal plane at a fixed vertical distance, this scanning mode is called constant-height mode. The quantitative height of the surface can be calculated by dividing $U_{F E S}$ by the slope of the linear region. However, constant-height mode can only provide quantitative height measurement for samples of a uniform material. Accurate height measurement is not available on a surface consisting of multiple materials. In the complex sample shown in figure 1(c), $C$ and $D$ designate positions with low and high reflectivity levels, respectively. In figure 1 (d), $U_{C}$ and $U_{D}$ represent $U_{F E S}$ measured at positions $C$ and $D$, and the S-curves at $C$ and $D$ are indicated by solid and dashed lines, respectively. Due to a higher reflectivity at position $D$, the dashed line has a larger slope than the solid line at position $C$. This reflectivity effect causes a larger $U_{F E S}$ difference between $U_{C}$ and $U_{D}$. Because $U_{F E S}$ is coupled with both surface height and reflectivity, the accurate height cannot be determined using constant-height mode in the current systems $[15,19,20]$.

(a)

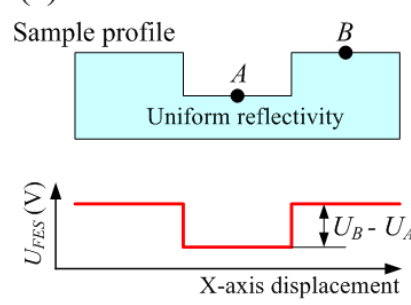

(c)

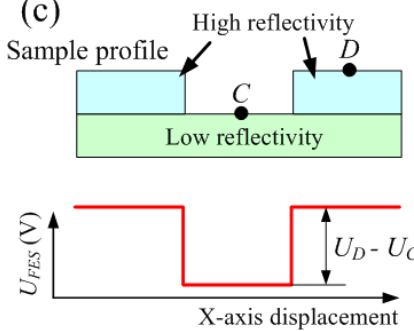

(d)
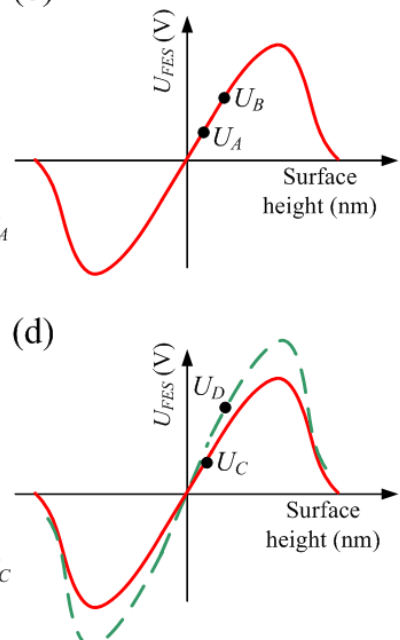

Figure 1. (a) Height and $U_{F E S}$ profiles and (b) S-curve on a uniform surface; (c) height and $U_{F E S}$ profiles and (d) S-curves on a nonuniform surface.
This study aimed to achieve quantitative height measurement for complex surfaces. We proposed a novel scanning method called z-axis modulation to decouple the surface reflectivity and morphology. Moreover, a calculation program and a homemade astigmatic optical profilometer were developed. The experimental results indicated that the proposed method can realize quantitative height measurement on a standard sample consisting of two different materials. Furthermore, the surface reflectivity can also be imaged through calculating the slope of the S-curves.

\section{Method}

Figure 2(a) illustrates the scanning trajectory of the proposed z-axis modulation mode. Scanning of the xy-axes is used to image the surface, and further z-axis movement produces an S-curve at every pixel in the image. Once a single S-curve has been obtained, the focused laser moves to the next point and measures another S-curve. This process repeats until S-curves on all the pixels have been captured. Figure 2(b) illustrates the method of calculating quantitative height from S-curves. The structural height difference shifts the S-curve in the $\mathrm{z}$-axis direction. Despite surface reflectivity affecting the slope and peak-to-peak voltage of each S-curve, the surface height can be extracted by determining the z-axis positions of the focal points, as indicated by the dashed line. Moreover, the slope of each Scurve can be used to evaluate surface reflectivity. In constant-height mode, the maximum measurable range of the sample height is based on the linear region of the S-curve, which is mainly determined by the numerical aperture (NA) of the objective lens. Using a high-NA lens can increase the measurable height. However, a trade-off exists between resolution and measurable range. In z-axis modulation, the measurable range is limited only by the travel distance of the z-axis scanner. Furthermore, multiple features may appear in the S-curve if the sample has a semitransparent surface coating; therefore, the thicknesses of multiple semitransparent layers could be measured. The main drawback of z-axis modulation is that the additional z-axis movement is time-consuming. Large data sets also require time for data acquisition and analysis.
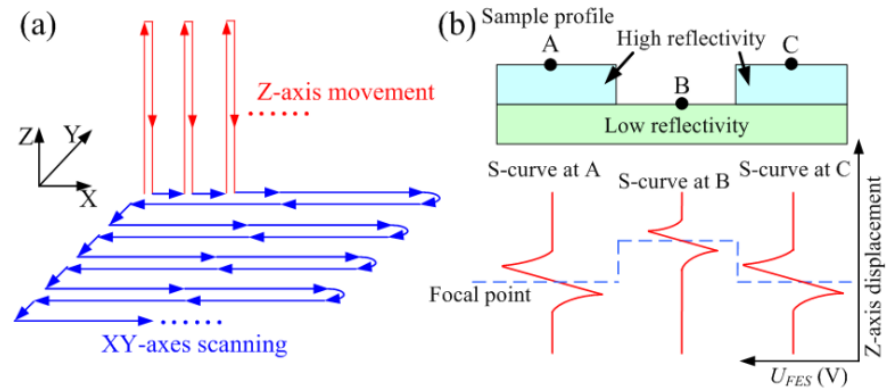

Figure 2. (a) Scanning trajectory and (b) height calculation method of z-axis modulation. 


\section{Instrumentation}

Figure 3(a) and (b) show the system configuration and a photograph of the homemade astigmatic optical profilometer, respectively. A commercial DVD pickup head (TOP1100s, TopRay Technologies) was adapted for the ADS. A polycarbonate chip was placed between the pickup head and the sample to enhance $U_{F E S}$. A customized amplifier (Highspeed Topray driver v122, Stromlinet Nano) was used to magnify small signals from the PDIC and generate $U_{F E S \text {. A }}$ control system was programmed using LabVIEW software to capture $U_{F E S}$ and generate the driving signals for scanning the xyz-axes. The control system consisted of a chassis (PXIe-1062Q, National Instruments), a real-time controller (PXIe-8840, National Instruments), and two fieldprogrammable gate array (FPGA) modules (PXIe-7961R and NI5781, National Instruments). The voltage range of the driving signals was adjusted to fit a scanner controller (E664.S3 piezo controller, Physik Instrumente) using a homemade amplifier. A closed-loop piezoelectric scanner (P611.3S NanoCube, Physik Instrumente) was placed under the sample to provide a maximum scan range of $100 \mu \mathrm{m}$ on the three axes. Although the control system can perform highspeed scanning, the actual speed is limited by the piezoelectric scanner's bandwidth. In the experiment, the zaxis movement of the scanner operated in an open loop to increase the scanning speed. A velocity of $1 \mathrm{~mm} / \mathrm{s}$ and a travel range of $41 \mu \mathrm{m}$ were used for z-axis scanning.

(a)

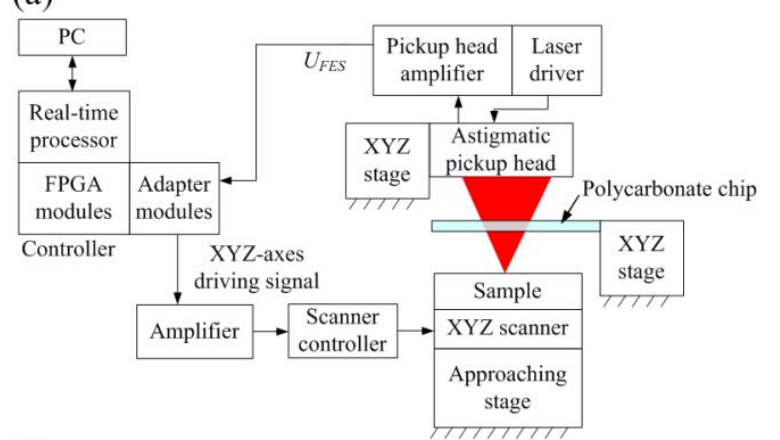

(b)

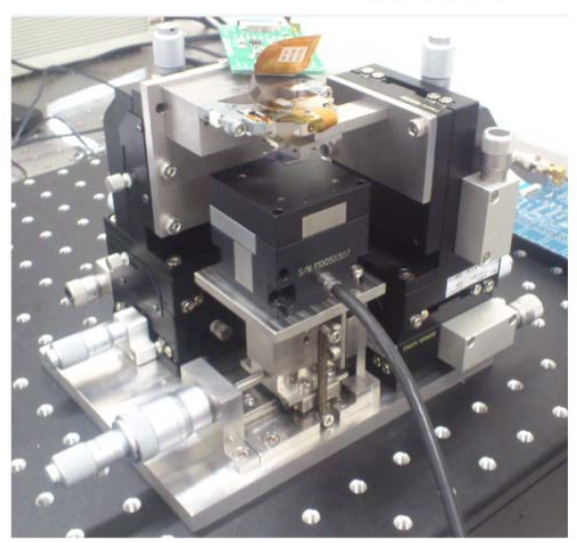

Figure 3. (a) Scheme and (b) photograph of the homemade astigmatic optical profilometer.
The $U_{F E S}$ data were temporarily stored in the control system during scanning and then transferred to a personal computer for offline analysis. The calculation method was programmed using MATLAB software. Figure 4(a) shows the flowchart for the height and slope calculation. First, the $U_{F E S}$ data of the first scan line were loaded, and the DC offset of the S-curve was removed. For each S-curve, linear curve fitting was performed to determine the slope and z-axis position of the focal point $\left(U_{F E S}=0\right)$. This process was repeated until the end of the data set. Finally, the calculated height and slope images were plotted. For comparison with constant-height mode, $U_{F E S}$ at arbitrary z-axis positions can also be extracted from the same data. As shown in figure 4(b), $U_{F E S}$ at three different z-axis positions $\left(z_{1}, z_{2}\right.$, and $\left.z_{3}\right)$ was imaged to obtain results around the focal point. Notably, a larger z-axis position represents a closer distance between the pickup head and sample. (a)

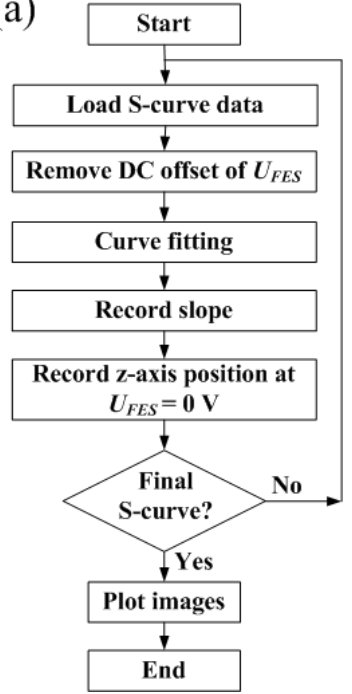

(b)

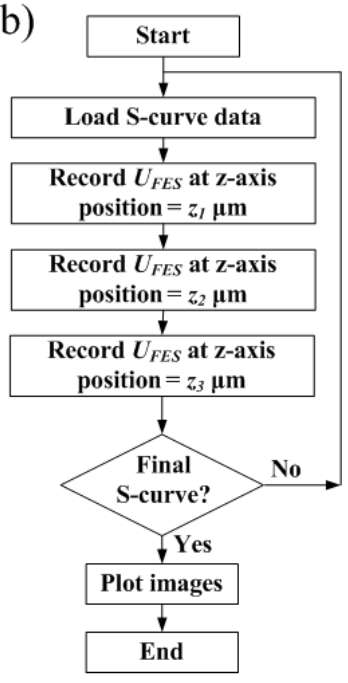

Figure 4. Calculation flowcharts for (a) height and reflectivity and (b) $U_{F E S}$ at certain z-axis positions.

\section{Results and discussion}

To verify the proposed method, a standard sample (R2L2S1N1, Thorlabs) was measured using the homemade astigmatic optical profilometer. The sample had a patterned chrome layer coated on a glass substrate. The thickness of the chrome layer was $120 \mathrm{~nm}$, according to the specification. First, the results of constant-height mode were examined using the calculation procedure in figure 4(b). The $U_{F E S}$ images at the chosen z-axis positions of 19.8, 21.1, and 22.4 $\mu \mathrm{m}$ are shown in figure 5(a), (b), and (c), respectively. The middle area in the images is the glass substrate, surrounded by the chrome layer. The three $U_{F E S}$ images show various contrasts between the two materials. As presented in figure 5(a), $U_{F E S}$ on the chrome layer was lower than that on the glass substrate. The $U_{F E S}$ contrast gradually decreased with increasing z-axis displacement and eventually inverted, as 


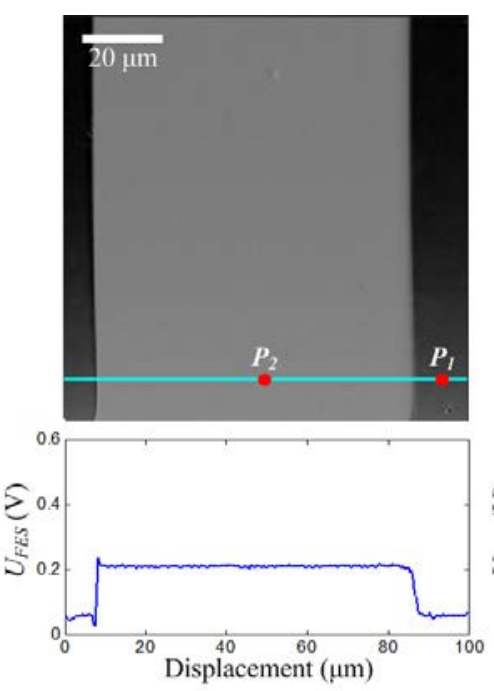

(a)
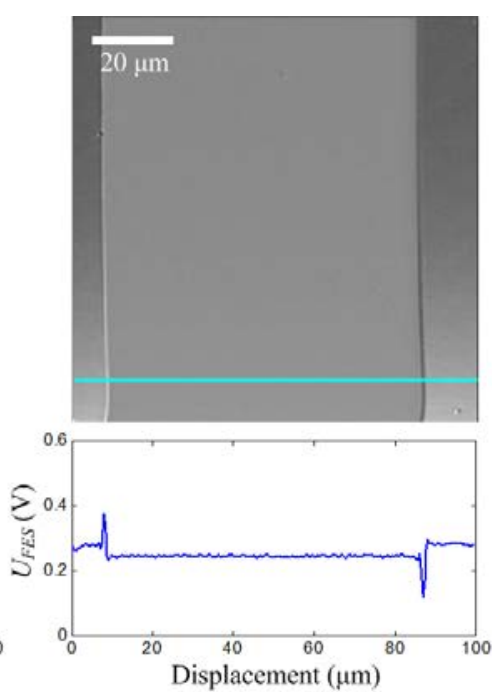

(b)

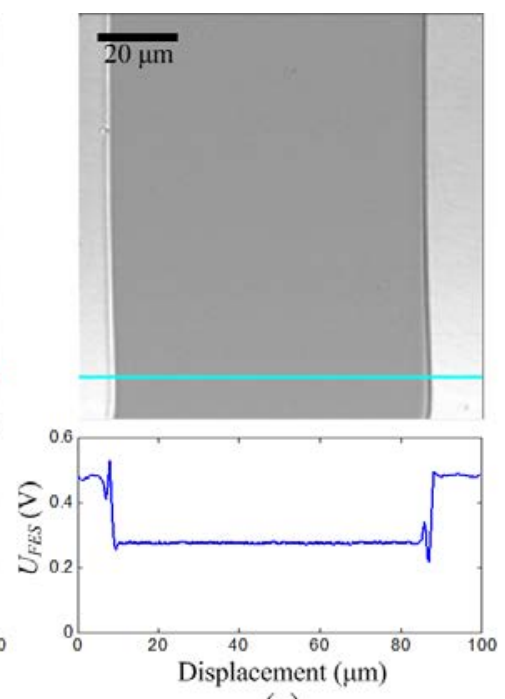

(c)

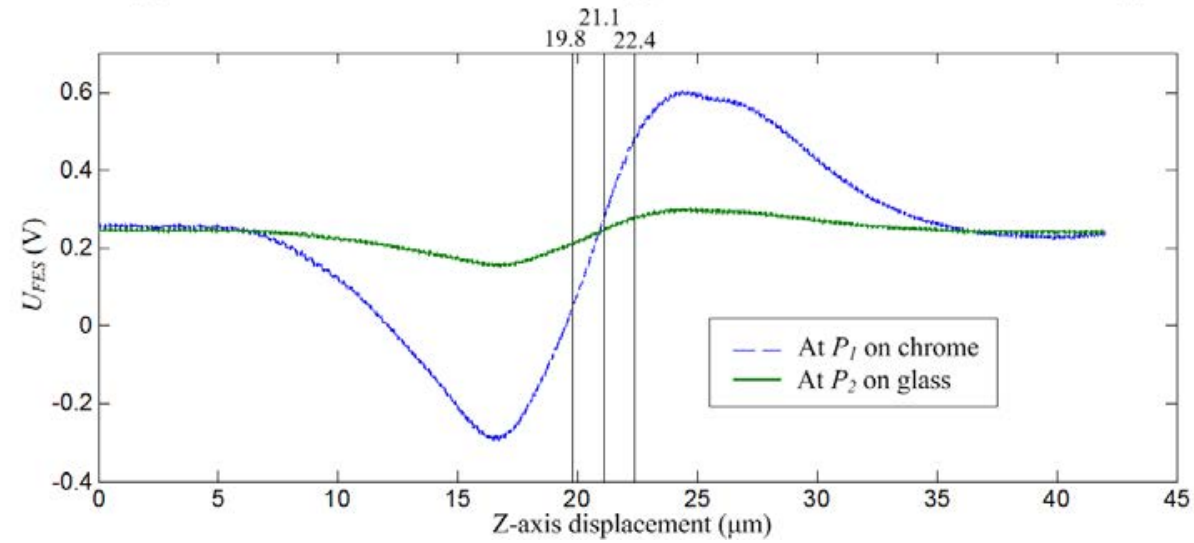

Figure 5. Focus error signal images at z-axis displacements of (a) $19.8 \mu \mathrm{m}$, (b) $21.1 \mu \mathrm{m}$, and (c) $22.4 \mu \mathrm{m}$; (d) S-curves on chrome and glass surfaces.

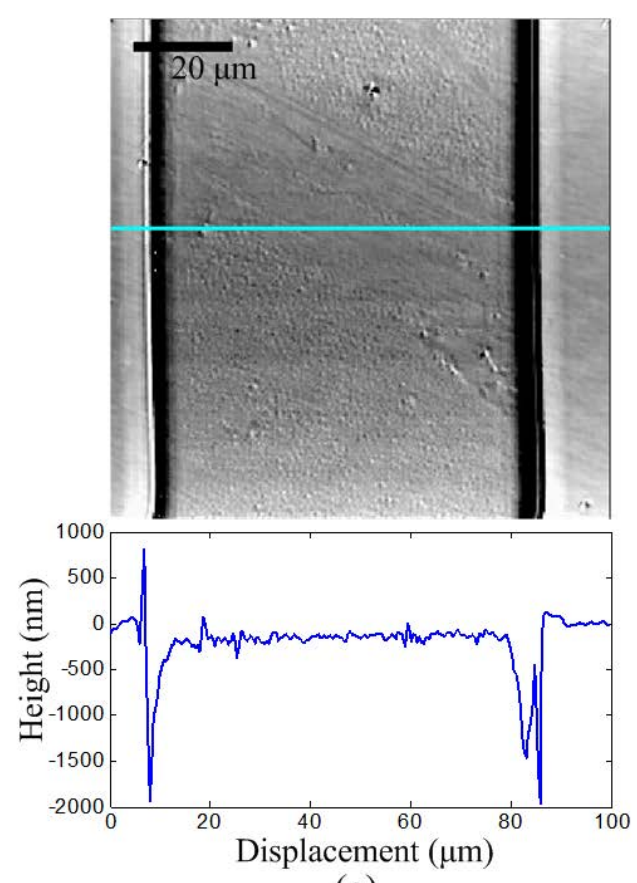

(a)

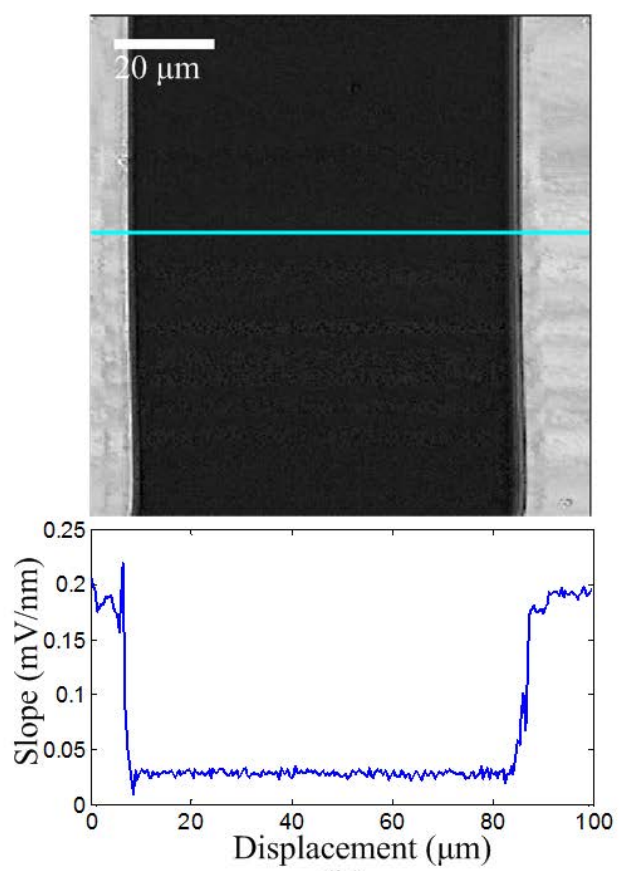

(b)

Figure 6. (a) Height image and (b) slope image 
shown in figure 5(b). As illustrated in figure 5(c), the chrome layer had a higher $U_{F E S}$ than the glass substrate. To explain this phenomenon, the S-curves measured on the two materials are compared in figure 5(d). The dashed line and solid line represent the S-curves at positions $P_{1}$ and $P_{2}$ in figure 5(a), respectively. Due to a higher reflectivity level on the chrome layer, the dashed line is shown to have a higher slope and peak-to-peak value than that of the glass substrate. The three vertical lines denote the z-axis positions of 19.8, 21.1, and $22.4 \mu \mathrm{m}$ used in figure 5(a), (b), and (c), respectively. The large slope at $P_{1}$ caused rapid variation of $U_{F E S}$ and resulted in the inverse contrast shown in figure 5(a) and (c). This result demonstrates that both the height and surface reflectivity affected $U_{F E S}$. Therefore, the quantitative height was not available in constant-height mode. We also noted that the S-curves were not in perfect symmetry due to the laser alignment and sample tilt, and we observed a DC bias of approximately $0.2 \mathrm{~V}$.

Figure 6(a) and (b) show the height and slope images, respectively, calculated using the procedure in figure 4(a). Figure 6(a) shows a step height of $126 \pm 18 \mathrm{~nm}$ (mean \pm STD), which is close to the $120 \mathrm{~nm}$ in the specification. Furthermore, small defects and particles on the surface were resolved clearly. However, sharp notches appeared on the edges between the two materials. This artifact was caused by an abnormal laser path due to the step geometry. Figure 6(b) shows a larger slope for the chrome layer than that for the glass substrate. The average slope values on chrome and glass were $0.186 \pm 0.005$ and $0.028 \pm 0.008 \mathrm{mV} / \mathrm{nm}$, respectively. Small surface defects on the glass in figure 6(a) are not visible in the slope image in figure 6(b). The slope image is mainly dominated by surface reflectivity. These results indicate that the proposed method can successfully decouple information on height and reflectivity.

\section{Conclusion}

This paper proposes z-axis modulation for quantitative height measurements on a surface made of different materials. The experimental results demonstrate that $\mathrm{z}$-axis modulation achieved accurate height measurements on a standard sample consisting of both chrome and glass materials. Apart from the observed structure height, local reflectivity on the surface was also imaged by calculating the slope from the S-curve. Moreover, compared with constantheight mode, the proposed method has a larger measurable range in the $\mathrm{z}$-axis direction. Furthermore, modifying the analytical method may enable the measurement of the thickness of multiple semitransparent layers. However, the additional z-axis movement in the proposed method was observed to reduce the imaging speed. To remedy this drawback, a high-speed z-axis scanner is required.

\section{Acknowledgements}

This research was supported by the Ministry of Science and Technology of Taiwan (MOST 106-2221-E-002-131).

\section{References}

[1] Lemaster R L and Beall F C 1996 The use of an optical profilometer to measure surface roughness in medium density fiberboard Forest Prod. J. 46 73-78

[2] Maccari A and Montecchi M 2007 An optical profilometer for the characterisation of parabolic trough solar concentrators Sol. Energy 81 185-194

[3] Haldal S, Shashikala, Rajesh P and Ali S S 2013 Quantitative Assessment of the Surface Roughness of Two Esthetic Restorative Materials after Tooth Brush Abrasion Using 3-D Profilometer and Scanning Electron Microscope Int. J. Health Sci. Res. 3 43-49

[4] Poon C Y and Bhushan B 1995 Comparison of surface roughness measurements by stylus profiler, AFM and noncontact optical profiler Wear 190 76-88

[5] Wennerberg A, Ohlsson R, Rosén B G and Andersson B 1996 Characterizing three-dimensional topography of engineering and biomaterial surfaces by confocal laser scanning and stylus techniques Med. Eng. Phys. 18 548-556

[6] Merola M, Ruggiero A, De Mattia J S and Affatato S 2016 On the tribological behavior of retrieved hip femoral heads affected by metallic debris. A comparative investigation by stylus and optical profilometer for a new roughness measurement protocol Measurement 90 365-371

[7] Morrison E 1995 A prototype scanning stylus profilometer for rapid measurement of small surface areas Int. J. Mach. Tool. Мапи. 35 325-331

[8] Lee D H and Cho N G 2012 Assessment of surface profile data acquired by a stylus profilometer Meas. Sci. Technol. 23105601

[9] Chang W C, Lai W C, Liao H S, Wang S C, Ho M S, Huang K Y, Chen L J and Chang C S 2012 Orientation-dependent friction between a carbon nanotube and graphite Appl. Phys. Lett. 101 063109

[10] Kumar N, Gautam G, Gautam R K, Mohan A and Mohan S 2016 Wear, friction and profilometer studies of insitu AA5052/ZrB 2 composites Tribol. Int. 97 313-326

[11] Sherrington I and Smith E H 1988 Modern measurement techniques in surface metrology: part II; optical instruments Wear 125 289-308

[12] Deck L and Groot de P 1994 High-speed noncontact profiler based on scanning white-light interferometry Appl. optics 33 7334-7338

[13] Ang K T, Fang Z P and Tay A 2014 Note: development of high speed confocal 3D profilometer Rev. Sci. Instrum. 85116103

[14] Groot de P 2015 Principles of interference microscopy for the measurement of surface topography Adv. Opt.s Photonics 71

[15] Hwu E T, Illers H, Jusko L and Danzebrink H U 2009 A hybrid scanning probe microscope (SPM) module based on a DVD optical head Meas. Sci. Technol. 20084005

[16] Hwu E T, Hung S K, Yang C W, Huang K Y and Hwang I S 2008 Real-time detection of linear and angular displacements with a modified DVD optical head Nanotechnology 19115501

[17] Liao H S, Chen Y H, Ding R F, Huang H F, Wang W M, Hwu E T, Huang K Y, Chang C S and Hwang I S 2014 High-speed 
atomic force microscope based on an astigmatic detection system Rev. Sci. Instrum. 85103710

[18] Ehrmann K, Ho A and Schindhelm K 1998 A 3D optical profilometer using a compact disc reading head Meas. Sci. Technol. 91259

[19] Liao H S, Huang K Y, Hwang I S, Chang T J, Hsiao W W, Lin H H, Hwu E T and Chang C S 2013 Operation of astigmaticdetection atomic force microscopy in liquid environments Rev. Sci. Instrum. 84103709

[20] Hsiao W W, Liao H S, Lin H H, Lee Y L, Fan C K, Liao C W, Lin P Y, Hwu E T and Chang C S 2013 Biophysical analysis of astrocytes apoptosis triggered by larval E/S antigen from cerebral toxocarosis-causing pathogen Toxocara canis Anal. Sci. 29 885-892 